Itinéraires Itinéraires

Littérature, textes, cultures

\title{
Représentations des intimités amoureuses dans la France du $\mathrm{Xx}^{\mathrm{e}}$ siècle
}

\section{Anne-Claire Rebreyend}

\section{(2penEdition}

\section{Journals}

Édition électronique

URL : http://journals.openedition.org/itineraires/1188

DOI : $10.4000 /$ itineraires. 1188

ISSN : 2427-920X

Éditeur

Pléiade

Édition imprimée

Date de publication : 1 décembre 2009

Pagination : 149-161

ISBN : 978-2-296-10791-5

ISSN : $2100-1340$

Référence électronique

Anne-Claire Rebreyend, "Représentations des intimités amoureuses dans la France du Xxe siècle », Itinéraires [En ligne], 2009-4 | 2009, mis en ligne le 02 septembre 2014, consulté le 03 mai 2019. URL: http://journals.openedition.org/itineraires/1188; DOI : 10.4000/itineraires.1188

Ce document a été généré automatiquement le 3 mai 2019.

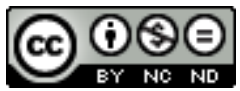

Itinéraires est mis à disposition selon les termes de la licence Creative Commons Attribution - Pas d'Utilisation Commerciale - Pas de Modification 4.0 International. 


\title{
Représentations des intimités amoureuses dans la France du $\mathrm{xx}^{\mathrm{e}}$ siècle
}

\author{
Anne-Claire Rebreyend
}

$1 \mathrm{Au}$ XIX ${ }^{\mathrm{e}}$ siècle, l'usage de l'adjectif « intime » prend de l'ampleur dans le domaine de la vie privée restreinte à l'individu ou au couple ${ }^{1}$. Ainsi, les «parties intimes » sont les organes sexuels, l'« hygiène intime » concerne les parties dissimulées du corps. Dans la seconde moitié du xx siècle, l'« union intime » ou les « relations intimes » ont le sens de rapports sexuels. Si le terme intime admet d'autres acceptions, on s'en tiendra ici aux intimités amoureuses. Il s'agit donc d'appréhender les représentations mouvantes du sentiment amoureux et de la sexualité pour écrire une histoire de l'intime².

2 L'histoire de l'intime est récente en France : d'une part, elle a longtemps été considérée comme un domaine futile au regard de l'histoire politique, économique ou sociale, un sujet anecdotique en histoire culturelle, d'autre part elle souffrait de la destruction et du silence des sources. Pourtant, on peut accéder au cœur de l'intimité amoureuse des individus grâce aux archives autobiographiques. À l'aide de ces sources, il est possible d'explorer les frémissements du cœur et les élans du corps des individus, en distinguant trois grandes périodes : l'intime feutré durant l'entre-deux-guerres, l'intime questionné de 1939 à 1965 et l'intime exhibé de 1965 à $1975^{3}$.

\section{Les perceptions de l'histoire de l'intime ou l'historien mis à nu}

3 L'histoire de l'intime doit beaucoup à l'histoire de la vie privée qui s'intéresse, à partir des années 1970, aux individus, à leurs représentations et à leurs émotions, à leurs manières de faire, de vivre, de sentir et d'aimer au quotidien. Les rapports du public et du privé, du collectif et de l'individuel, du masculin et du féminin, du spectaculaire et de l'intime, suscitent des interprétations diverses que rappelle Michelle Perrot ${ }^{4}$. Scrutant les traités 
de civilité depuis le $\mathrm{xVI}^{\mathrm{e}}$ siècle, Norbert Élias montre comment un affinement de la sensibilité nommé "pudeur» conduit certains actes (se moucher, déféquer, avoir des relations sexuelles) autrefois accomplis en public, à se privatiser. Jürgen Habermas et Richard Sennett indiquent une décadence des sociabilités à l'époque des Lumières par l'empiètement des États pour le premier, par l'enfermement dans la famille nucléaire pour le second. Philippe Ariès relève également le passage d'une sociabilité anonyme, où les notions du privé et de public sont confondues, à une sociabilité éclatée en domaines bien définis où la vie domestique prend de plus en plus d'importance. Le XIX siècle constitue l'âge d'or du privé, tandis que se poursuit au $\mathrm{xx}^{\mathrm{e}}$ siècle un processus de sectorisation par catégories d'âge, de sexe, d'ethnie ou de région. La face externe et publique de la vie privée se différencie de sa face interne et cachée : l'intime constitue alors le « privé du privé » selon l'expression d'Alain Corbin 5 .

4 L'histoire de l'intime est donc une histoire de l'indicible, de l'enfoui - une histoire du secret $^{6}$ qui se trame au plus profond des êtres, et devant laquelle les historiens et les historiennes peuvent éprouver des scrupules. Le lecteur de Lettres perdues peut être partagé entre «le droit de regard et le sentiment de violer un territoire propre", soulignent Philippe Artières et Jean-François Laé7. Marc Bloch lui-même identifiait l'historien à un ogre dont l'écriture se nourrit de chair8. Le fait d'entrer dans l'intimité gêne et fascine également les sociologues. Didier Le Gall propose le terme de "scopophilie» pour désigner le chercheur en sciences sociales qui prend l'intimité comme objet d'observation. D'autres sociologues prononcent le terme de voyeurisme, pour s'en défendre comme Michel Bozon, ou pour le revendiquer tel Daniel Welzer-Lang, qui prône une méthode " d'observation participante" ".

5 Le problème de la réception des travaux sur l'intime est inévitable. L'étude d'une pratique réprouvée ou moquée par la société nécessite un arsenal de précautions pour écarter le soupçon de grivoiserie ou de désinvolture, comme chez Patrick Baudry dans La Pornographie et ses images ou chez Thomas Laqueur dans Le Sexe en solitair ${ }^{10}$. L'autre écueil est celui de la subjectivité auquel les historiens et les historiennes ne sauraient échapper. Depuis quelques années, à l'instar d'Alain Corbin, promu «historien du sensible » ou d'Ariette Farge, qui défend une approche émotionnelle des textes, les historiens s'inspirent des méthodes des anthropologues et des sociologues sur l'intime ${ }^{11}$. La question de la subjectivité en histoire et de la place du « je » dans le récit historique est clairement posée par le renvoi de l'histoire de l'intime au vécu propre de l'historien ou de l'historienne. Mais ceci peut aussi devenir un atout en appelant à l'imagination et aux " archives intérieures ${ }^{12}$ » pour tenter d'appréhender les émotions et les rêves des individus du passé, tout en prenant garde de ne pas substituer sa voix aux leurs.

\section{Les archives autobiographiques au cœur de l'intime}

Pour accéder à l'intimité amoureuse et sexuelle des Français du $\mathrm{xx}^{\mathrm{e}}$ siècle, les archives autobiographiques, qui rassemblent les récits autobiographiques écrits ou oraux, les journaux personnels et les correspondances, sont d'une grande richesse ${ }^{13}$. Qualifiées aussi d'archives personnelles, de sources du moi ou d'egodocuments, elles forment un « immense et presque indélimitable continent ${ }^{14}$ » qui n'a cessé de questionner l'Histoire. Longtemps négligées à la fois pour des raisons matérielles (difficultés d'accès aux fonds privés) et subjectives (méfiance des historiens et des historiennes pour ces écritures ordinaires), les archives autobiographiques sont devenues plus largement accessibles 
grâce à la mise en place de fonds d'archives comme celui de l'APA (Association pour l'autobiographie et le patrimoine autobiographique ${ }^{15}$ ). Ces sources offrent un regard neuf sur les représentations sexuelles et amoureuses et permettent de brosser de longs portraits individuels qui donnent corps à l'Histoire.

Quelques précautions méthodologiques s'imposent lorsqu'on s'engage dans l'univers autobiographique. Selon Philippe Lejeune, l'existence d'un «pacte autobiographique » est caractéristique du genre : l'auteur s'engage à raconter tout ou partie de sa vie dans un esprit de vérité, et demande au lecteur de le croire, mais également de l'aimer et de l'approuver. Le pacte est donc « référentiel » et " relationnel ${ }^{16}$ ». En dehors de ce critère commun, les archives autobiographiques se distinguent par leur diversité. Le temps de l'écriture et celui du vécu ne sont pas identiques dans le récit autobiographique, œuvre de longue haleine durant laquelle l'auteur réfléchit au sens qu'il veut donner à sa vie, et dans le journal intime, souvent écrit à chaud ${ }^{17}$. Nonobstant, maints écrivains furent autobiographes et diaristes (Stendhal, Sand, Gide, Leiris...), et journal et autobiographie parfois se mêlent et se complètent. Certains récits autobiographiques mettent en œuvre un va-et-vient du passé au présent, comme certains journaux glissent vers l'écriture synthétique et la rétrospection d'assez longue portée. Il arrive même que le diariste se relise et ajoute des commentaires en marge ou entre les lignes.

D'autre part, le récit autobiographique vise une certaine publicité, au moins familiale (écrit pour les enfants et/ou les petits-enfants, voire pour les lecteurs de l'APA), tandis qu'un journal intime, retrouvé par hasard dans un marché aux puces ou un grenier, était voué à rester secret. Mais si le contenu d'un récit autobiographique est généralement moins intime que celui d'un journal, il ne faut pas sous-estimer la force de l'autocensure dans les journaux, ni oublier que depuis la fin du xix ${ }^{e}$ siècle et « l'indiscrétion des frères Goncourt ${ }^{18}$ ", tous les écrivains qui tiennent un journal ont une publication possible à l'esprit. Rien n'empêche donc le diariste anonyme d'en rêver aussi.

Dans les correspondances, il ne s'agit plus d'un rapport solitaire à l'écrit, mais d'un dialogue avec l'être aimé ou avec une personne promue spécialiste du cœur comme la philosophe Simone de Beauvoir, la journaliste Menie Grégoire ou les médecins du MFPF (Mouvement français pour le Planning familial ${ }^{19}$ ). Dans ce dernier cas, les correspondants se présentent, donnent des informations sur leur cadre de vie, avant d'énoncer leur problème amoureux ou sexuel. Certaines lettres, longues et détaillées, s'apparentent à un récit autobiographique. Les correspondances amoureuses suscitent plus de difficultés ${ }^{20}$, mais fournissent des éléments intimes passés sous silence dans une autobiographie (trop publique) ou dans un journal (trop personnel).

Les historiens et les historiennes, plus tolérants vis-à-vis du témoignage oral ou du journal personnel, se méfient de la vision reconstruite du vécu proposée par l'autobiographe ${ }^{21}$. Celui-ci est d'autant plus soupçonné de cacher ou d'altérer la vérité que l'on touche au domaine sexuel : les archives autobiographiques ne rendraient pas compte du vécu sexuel des personnes, mais de leurs fantasmes; de toute façon, les individus en disent toujours trop ou pas assez.

11 Pourtant les archives autobiographiques constituent des sources pour l'Histoire qui ne sont pas nécessairement plus mensongères que les autres. En les croisant avec des documents de nature différente, il est possible sinon de les authentifier, du moins de détecter les inventions trop flagrantes. En se livrant à une critique historique (comme pour n'importe quelle autre source), on peut appréhender ce que George Perec appelle 
«l'infraordinaire », des informations sur des faits quotidiens et minuscules introuvables ailleurs ${ }^{22}$. Enfin, ce n'est pas tant une improbable vérité unique qu'il s'agit de saisir que les représentations individuelles et collectives de la sexualité et de l'amour.

Les archives autobiographiques suscitent une double approche: elles apportent d'une part des éléments sur l'évolution des représentations et des pratiques amoureuses et sexuelles, et elles donnent, d'autre part, l'occasion d'identifier les façons d'écrire et de dire l'intime.

\section{L'intime feutré durant l'entre-deux-guerres}

13 Les auteurs et les auteures d'archives autobiographiques qui sont adultes durant l'entredeux-guerres font preuve d'une grande discrétion. Celle-ci peut s'expliquer par la difficulté de trouver les mots pour dire l'intime-difficulté redoublée par une autocensure à cause de la crainte d'être lu (dans le cas du journal) ou de la certitude d'être lu (pour les lettres et les récits autobiographiques). La pudeur des écrits suggère aussi une pudeur au quotidien qui se retrouve aussi bien chez des catholiques fervents pétris de culpabilité face au sexe ${ }^{23}$ que chez certains de leurs contemporains aux mœurs plus libres. Il n'est jamais question de « sexualité » ou de « sexe » dans les textes, mais de " vie sexuelle », de « sensualité », ou simplement de « la chose ».

14 La sexualité reste difficile à dire, de même que tout ce qui est lié au corps, à la contraception et à l'avortement. En effet, mentionner des pratiques anticonceptionnelles, c'est déjà parler de sexualité à une époque où le retrait et l'abstinence périodique sont les moyens de contraception les plus courants. De ce fait, il s'avère moins gênant pour un autobiographe ou une autobiographe d'inventorier le nombre d'enfants et de présenter la sexualité de manière fonctionnelle, en tant que moyen permettant de produire les générations futures. La sexualité est donc située du côté de la nature et de l'instinct de reproduction, mais en dehors de la culture et de la recherche du plaisir pour lui-même. Si dans les faits, les couples tentent de limiter les naissances et recherchent le plaisir, la sexualité n'apparaît souvent dans leurs discours que sous l'angle «naturel» de la reproduction. Cette opposition entre une sexualité liée à la nature et une sexualité modifiée par la culture sera omniprésente dans les débats ultérieurs sur la contraception et l'avortement après la Seconde Guerre mondiale.

15 Si la sexualité est tue, l'amour est au centre des discours bien qu'il s'agisse en réalité d'une sorte bien particulière d'amour: l'amour conjugal. Les hommes et les femmes adultes de l'entre-deux-guerres accordent une place fondamentale au mariage d'amour, basé sur le sentiment amoureux et donnant droit à la sexualité. La volonté de choisir son/ sa partenaire afin de vivre un bonheur à deux pour la vie est forte, même si elle ne résiste pas toujours au quotidien. Si la majorité des auteurs et des auteures insistent sur les liens amoureux, il est probable que nombre de mariages dits d'amour ne diffèrent guère de mariages de raison réussis car la focalisation sur la notion d'amour cache bien d'autres raisons de convoler. Pour les femmes, le mariage apporte un prestige social indéniable à une époque où les célibataires sont à la fois plaintes et mal vues, et il permet d'avoir des enfants légitimes. L'institution conjugale offre les mêmes avantages aux hommes à qui la division tranchée des rôles au sein du ménage permet, en outre, de se consacrer pleinement à leur carrière. Enfin, la grande affaire du mariage n'est pas tant l'amour que la sexualité : le mariage est le cadre normal de la vie sexuelle à une époque où la sexualité hétérosexuelle non conjugale est considérée en termes de danger (maladies vénériennes) 
et de déshonneur (grossesses illégitimes). Se marier, c'est donc avoir un (ou une partenaire) régulier, et en principe toujours consentant puisque le viol conjugal n'est pas reconnu par la loi. La multiplication des discours sur l'amour fait donc contrepoint à l'absence de discours sur la sexualité : quand les individus parlent d'amour à l'horizon du mariage, ils englobent aussi le désir sexuel, l'attraction physique, le plaisir des corps. Mais cet aspect de l'amour et du mariage n'est pas formulé, notamment chez les femmes.

Les personnes qui vivent leur enfance et leur jeunesse dans l'entre-deux-guerres sont plus attentives à l'éveil de leur corps et à la montée du désir, même si leur plume reste pareillement discrète. Les jeunes des deux sexes rêvent de la sexualité, mais redoutent l'acte sexuel pour des raisons différentes: les filles ont la " peur au ventre ${ }^{24}$ ", tandis que les garçons craignent de ne pas être à la hauteur (certains se souciant aussi du risque de grossesse). L'amour et la sexualité semblent faire partie de deux mondes différents dans l'esprit des jeunes gens, tant les interdits sur la sexualité sont forts pour les filles et l'initiation sexuelle des garçons (par des prostituées ou des femmes mariées) dépourvue de sentiment. L'homosexualité apparaît peu dans les archives autobiographiques de cette époque $^{25}$. Cela reflète-t-il une société qui a sacralisé l'amour hétérosexuel car l'hétérosexualité était inévitable pour remédier au problème de la dépopulation ${ }^{26}$ ? L'entre-deux-guerres marque le triomphe d'une certaine idée de l'hétérosexualité en tant que sexualité et culture dominante : l'insistance des individus sur le mariage d'amour, la mise en valeur du lien entre sexualité et reproduction et l'absence d'une réflexion sur la contraception et l'avortement vont dans ce sens.

L'entre-deux-guerres s'apparente à une époque de transition entre le murmure amoureux des adultes et la volonté de savoir des plus jeunes, en particulier des jeunes femmes. Un certain nombre de questions qui sont à peine esquissées dans les archives autobiographiques traitant de l'entre-deux-guerres (le problème de la contraception et de l'avortement, la possibilité de vivre un amour homosexuel au grand jour, la sexualité juvénile pour les filles) seront posées avec âpreté dans les décennies suivantes, celles de l'intime questionné.

\section{L'intime questionné de 1939 à 1965}

Le contexte de la guerre et de l'occupation explique la création de nouvelles sources pour l'Histoire. En effet, l'angoisse de la mort, les privations et les frustrations au quotidien poussent les individus à s'interroger sur le contenu de leurs liens affectifs et de leur sexualité, dans des lettres, des journaux et des autobiographies toujours plus nombreux - sans compter les archives judiciaires où la sexualité est disséquée pour traquer les femmes de "mauvaise $v^{2}{ }^{27}$ ». Les époux séparés s'écrivent; la distance renforce souvent leurs liens, les délitant et les brisant parfois. Les soldats et les prisonniers ouvrent un journal de guerre et de captivité. Les femmes agissent de même sur les chemins de l'exode ou dans l'attente de nouvelles. Les amants noircissent des pages, renouvelant leur serment d'amour et exprimant leurs doutes et leurs angoisses face à l'avenir ${ }^{28}$. Les retraités et les retraitées d'aujourd'hui se souviennent de leur jeunesse bouleversée par le conflit mondial ${ }^{29}$. En ce sens, les années de guerre marquent le début d'un intime questionné.

Le questionnement sur l'intime se prolonge une fois la paix retrouvée par le surgissement d'une parole féminine sur la sexualité: les femmes adultes dénoncent leur malaise conjugal; les filles aspirent au plaisir par le jeu du flirt et de la séduction. L'amour 
conjugal mis en exergue durant l'entre-deux-guerres, est toujours au centre des préoccupations, mais il est désormais couplé avec la sexualité comme l'indique la popularité de l'expression "faire l'amour » pour désigner l'activité sexuelle. Il ne s'agit plus simplement de s'aimer pour former un couple heureux, mais de se prouver la force de cet amour en se donnant un plaisir sexuel réciproque. Les notions de devoir, de caractère moral, d'union spirituelle caractérisant le mariage dans les discours anciens, perdent du terrain au profit de l'idéal du "companionate marriage», défendu depuis l'entre-deux-guerres, et décliné dans les décennies suivantes sous la forme de l'amour fusionnel. Les femmes, surtout, rêvent d'un couple formé de deux individus parfaitement soudés qui communiquent leurs idées et leurs émotions. Cet intérêt croissant pour le dialogue entre époux correspond à un moment où le concept de communication est à la mode: il accompagne les nouvelles technologies de l'information, détermine la disposition des pièces dans les foyers modernes et fournit même le titre de la première revue structuraliste ${ }^{30}$.

Cependant, dans une société où les rôles des hommes et des femmes sont nettement distingués et opposés (valorisation de l'homme comme chef de famille et de la femme comme mère au foyer), la communication au sein du couple n'est pas toujours facile à établir dans le sens attendu par les femmes. En outre, leurs difficultés pour contrôler leur fertilité et s'approprier leur corps pèsent toujours lourdement sur la sexualité dans le mariage, comme en dehors. La peur de la grossesse empêche bien des femmes de partager les joies physiques avec leur conjoint et marque la rupture, voire l'échec de la sexualité comme langage de l'amour. Elle dissuade nombre de jeunes filles de passer à l'acte et hante les relations sexuelles juvéniles.

21 L'injonction à la communication amoureuse et sexuelle atteint surtout les classes moyennes et supérieures, dont les revenus, la sociabilité et le style de vie rendent possible cette focalisation sur le couple privé. Dans les classes populaires (et dans de nombreux ménages catholiques pratiquants, indépendamment du niveau de vie), les différences de genre sont plus marquées et mieux intégrées avec l'importance d'avoir un enfant rapidement comme preuve de fertilité pour les ouvriers et comme but premier du mariage pour les catholiques. L'effort fourni pour entretenir une famille nombreuse absorbe l'attention des époux, pour lesquels la question de la communication intime se pose de manière moins centrale.

22 Si l'amour et la sexualité sont célébrés dans le mariage, la sexualité reste suspecte en dehors lorsqu'elle concerne les femmes hétérosexuelles, tandis que l'homosexualité masculine est condamnée et l'homosexualité féminine ignorée. Par conséquent, les personnes ayant des pratiques hors mariage privilégient les sentiments dans leurs discours. Pour les jeunes, en particulier les filles, l'amour peut parfois excuser les pratiques sexuelles prénuptiales, d'où l'image plus positive de la «fille amoureuse ${ }^{31}$ ». Pour les amants, durant la guerre comme après, l'amour légitime la sexualité. Des hommes et des femmes homosexuels (surtout s'ils sont catholiques pratiquants) se réclament aussi de l'amour, même si la séparation entre sentiment et sexualité peut constituer un modèle alternatif pour certains. C'est enfin au nom de l'amour (entre époux, entre parents et enfants) que se développe le discours sur la séparation entre sexualité et procréation. Paradoxalement, c'est pour le même motif que certaines pratiques contraceptives (retrait, injection, abstinence périodique) sont ignorées ou rejetées par les amoureux. 

peut découler indirectement des bouleversements de la guerre. Les individus malmenés par le conflit aspirent à un idéal de vie conjugale et familiale. Parce qu'elle génère des frustrations sexuelles et affectives, la guerre stimule un questionnement sur l'intime. Les décennies suivantes n'apportent pas de réponses, mais entraînent d'autres questions : le mariage et la famille conduisent-ils à l'épanouissement individuel ? L'idéal de l'amour fusionnel, qui se substitue à la notion d'amour patiemment construit, est-il compatible avec la répartition des rôles masculin et féminin, avec le problème du contrôle des naissances, avec l'aspiration au plaisir féminin? La volonté de trouver des réponses conduit les individus à extérioriser toujours plus leur vie intime, inaugurant ainsi l'ère de l'intime exhibé.

\section{L'intime exhibé de 1965 à 1975}

Les années $1968^{32}$ sont associées dans la mémoire collective à la "libération sexuelle ", source d'épanouissement mais aussi de crispation. Les intuitions de Foucault et les analyses de Michel Bozon montrent comment se mettent en place des normes plus individualisées et intériorisées de la sexualité dans un contexte de médicalisation de celle-ci ${ }^{33}$. La revendication d'un droit au plaisir a exacerbé une souffrance liée à l'absence de plaisir. Plutôt que de "libération sexuelle" des individus, il vaut mieux parler de libération des discours sur la sexualité.

Les individus évoquent désormais autant la sexualité que l'amour dans les archives autobiographiques. Le nom sexualité lui-même et l'adjectif sexuel se sont démocratisés : on parle de «rapport sexuel», de "relation sexuelle». Le rôle des femmes dans cette montée en puissance de la parole sur l'intime se voit conforté. Désormais elles écrivent et parlent du sexe autant que les hommes et avec le même souci de détails si l'on en croit le volumineux courrier qu'elles adressent en leur nom propre ou en celui de leur partenaire aux médecins, journalistes, écrivains "spécialistes » de la sexualité. Longtemps, les femmes ont disposé de droit et de fait d'une moindre liberté sexuelle que les hommes. Ayant fraîchement conquis cette liberté, il n'est pas très étonnant qu'elles veuillent à tout prix la «normaliser » et la faire entrer dans le moule médical. Elles veulent autant éviter leur propre culpabilité que les débordements supposés ou réels de la sexualité masculine.

La «libération sexuelle » s'est traduite à la fois par un épanouissement sur le plan personnel et par une conscience aiguë des problèmes sexuels qui n'ont pas lieu d'être dans une sexualité considérée comme "normale ». Les individus des années 1970, en défendant l'idée d'un "droit au plaisir », ont aussi fabriqué une souffrance liée à l'absence de plaisir. Georges Vigarello a démontré que c'est en se démocratisant et en promettant le bien-être, que la beauté a très conjointement élaboré de l'épanouissement et de la crispation $^{34}$. Les travaux de David Le Breton indiquent que la fragilité du corps, sa vulnérabilité à la maladie, au vieillissement, sont devenues intolérables et qu'il devient nécessaire de le contrôler parfaitement pour être heureux ${ }^{35}$. N'en est-il pas de même avec l'idée de «libération sexuelle» pour tous, avec l'assignation au plaisir sexuel de la « révolution sexuelle », avec l'assimilation de la sexualité au bien-être et à la santé dans le monde médical ${ }^{36}$ ?

L'expression « droit au plaisir », qui revient aussi bien dans les slogans des féministes, que chez les tenants de la révolution sexuelle ou les sexologues, ne tranche pas la question de 
savoir si l'on revendique la possibilité de chercher son plaisir où l'on veut, ou bien la garantie de la jouissance elle-même. Elle ne dissipe pas plus l'ambiguïté du mot «droit ", entre une acception subjective (ce qui fonde une revendication) et une acception objective (une règle à laquelle on doit se conformer), tant ces différents discours sont producteurs de normes. Avec la désacralisation de la sexualité et la montée de l'érotisme, ces normes sont de plus en plus intériorisées. L'intime est exhibé, certes, mais selon des scripts et des images calibrés, bien définis, et difficiles à contester.

Quoi qu'il en soit, obtenir un orgasme n'est plus un droit réservé aux seuls hommes adultes hétérosexuels, mais il est revendiqué par les femmes. Les homosexuels et les jeunes des deux sexes en général se saisissent aussi de ce droit, pour dire que leur sexualité doit être acceptée. En même temps, il faut se conformer à cette règle du plaisir pour se sentir heureux, épanoui, en bonne santé. Par conséquent, l'individu qui n'y parvient pas peut y trouver un motif de plainte - plainte qui revient sans cesse dans les lettres, les journaux, les autobiographies.

La sexualité n'est plus considérée comme quelque chose de magique ou de mystérieux qui échapperait à l'emprise technique ou médicale. Le vocabulaire et les procédés de la technique et de l'industrie ont gagné le domaine de l'intime: la fonction sexuelle doit produire du plaisir, et il est possible de la réparer si ce n'est pas le cas. De ce fait, la pilule et le stérilet sont acceptés par les femmes et leurs partenaires, non seulement parce qu'ils sont fiables, mais parce qu'ils sont jugés confortables. De la même façon, les techniques des sexologues sont supposées apporter plus de plaisir, d'où la massification des pratiques sexuelles bucco-génitales ${ }^{37}$. Enfin, «l'orgasme » en général (et "l'orgasme simultané » en particulier), permet de mesurer la qualité d'une relation sexuelle. Le concept médical de « santé sexuelle ", développé par les sexologues et repris à son compte par l'OMS en 1975, signe la consécration de "l'optimisme sexuel ", en légitimant socialement le lien entre le bien-être et la sexualité, dans une représentation de la sexualité dans sa dimension érotique et non reproductive ${ }^{38}$.

De 1965 à 1975, la littérature et le cinéma érotique se démocratisent (en attendant le cinéma X), les chansons d'amour parlent aussi de sexe, les magazines à grand tirage s'emparent des questions sexuelles, la publicité érotise les comportements pour vendre n'importe quel produit de consommation, l'intimité des individus est analysée au cours d'émissions radiophoniques, ou plus rarement, de télévision. Tout cela n'aurait pas été possible sans la puissance des moyens de communication modernes et la création d'une culture de masse qui diffuse modèles et représentations sexuelles ${ }^{39}$.

C'est ainsi que la sexualité devient une science, une technique et le produit d'une consommation de masse. Une science, car son étude s'est considérablement développée dans les sciences expérimentales avec l'apparition de la nouvelle sexologie, et s'amorce dans les sciences humaines au cours de la deuxième moitié des années $1970^{40}$. Une technique, car il faut désormais bien connaitre la sexualité (vocabulaire, positions, caresses) pour la pratiquer du mieux possible, pour la programmer et la maîtriser (techniques du corps, contraception). Le produit d'une consommation de masse, enfin, avec des achats de manuels, de films et autres livres érotiques, des consultations médicales (gynécologiques ou sexologiques), la prise d'un contraceptif, dont on attend un gain de plaisir sexuel. Dans un monde où l'érotisme se diffuse massivement, il semble important d'en avoir sa part pour être heureux.

Faire l'histoire de l'intime, c'est démontrer que l'idée, longtemps ancrée, d'une sexualité et d'un sentiment amoureux anhistoriques, naturels et immuables, n'a plus lieu d'être. 
Les individus n'envisagent pas de la même façon l'amour au début des années 1920 et au milieu des années 1970. De même, leurs représentations de la sexualité, à savoir les pratiques sexuelles qu'ils jugent attractives ou répulsives, les mots qu'ils prononcent ou qu'ils censurent, les gestes qu'ils s'autorisent ou qu'ils proscrivent, varient au fil du $x^{e}$ siècle.

Cette histoire de l'intime se prête fort mal à la généralisation et ne doit être qu'extrêmement nuancée. On peut certes délimiter de grandes périodes historiques concernant l'intime et mettre en avant des ruptures et des changements dans les intimités amoureuses. Mais les frontières de ces grandes périodes demeurent poreuses; celles-ci se recoupent, s'entrecroisent et s'opposent parfois, à l'image des sentiments que ressentent les individus eux-mêmes.

\section{NOTES}

1. Voir Véronique Montémont, «Dans la jungle de l'intime: enquête lexicographique et lexicométrique (1606-2008) », supra.

2. Bien entendu, l'histoire de l'intime peut aussi concerner d'autres sentiments et émotions comme l'amitié, la pudeur, la joie, la tristesse, la peur... De même, le corps, l'imaginaire et les sensibilités relèvent de l'intime, ainsi que les lieux ou les objets qui rappellent les affections humaines. Citons, entre autres, les travaux d'Anne Vincent-Buffault, Daniel Roche, Jean-Claude Bologne, Georges Minois, Theodore Zeldin, Alain Corbin, Georges Vigarello.

3. Cette dernière partie reprend les principales conclusions de mon ouvrage Intimités amoureuses. France 1920-1975, Toulouse, Presses universitaires du Mirail, 2009.

4. Michelle Perrot, "Introduction ", dans Philippe Ariès et Georges Duby (dir.), Histoire de la vie privée, t. IV, De la Révolution à la Grande Guerre [1987], Paris, Seuil, 1999, p. 7-12.

5. Alain Corbin, Historien du sensible. Entretiens avec Gilles Heuré, Paris, La Découverte, 2000, p. 42.

6. Michel Foucault, Histoire de la sexualité, t. I, La volonté de savoir, Paris, Gallimard, 1976, p. 49.

7. Philippes Artières, Le Livre des vies coupables. Autobiographies de criminels (1896-1909), Paris, Albin Michel, 2000, p. 1 ; Philippe Artières, Jean-François Laé, Lettres perdues. Écriture, amour et solitude $X I X^{e}-X X^{e}$ siècles, Paris, Hachette Littératures, 2003, p. 256.

8. Marc Bloch, Apologie pour l'histoire ou métier d'historien [1941], Paris, Armand Colin, 1974, p. 35.

9. Didier Le Gall, « Sociologie, scopophilie et intimité », MANA, Revue de sociologie et d'anthropologie, $\mathrm{n}^{\circ} 3,1^{\mathrm{er}}$ semestre 1997 , p. 12-13; voir aussi la mise au point de Daniel Welzer-Lang, "L'échangisme: une multisexualité commerciale à forte domination masculine», Sociétés contemporaines, $\mathrm{n}^{\circ} 41-42,2001$, p. 113-114.

10. Patrick Baudry, La Pornographie et ses images, Paris, Armand Colin, 1997, p. 21-22; Thomas Laqueur, Le Sexe en solitaire. Contribution à l'histoire culturelle de la sexualité [2003], Paris, Gallimard, 2005, p. 21.

11. Citons les numéros de MANA, Revue de sociologie et d'anthropologie, «Approches sociales de l'intime » en 1997 et d'Ethnologie française, «Intimités sous surveillance » en 2002, ainsi que la traduction française du livre d'Anthony Giddens, La Transformation de l'intimité. Sexualité, amour et érolisme dans les sociétés modernes, Paris, Le Rouergue / Chambon, 2004.

12. Michel Lagrée cité par Alain Corbin, Historien du sensible, op. cit., p. 117. 
13. Il est également intéressant d'utiliser les archives judiciaires à l'instar d'Anne-Marie Sohn, Du premier baiser à l'alcôve. La sexualité des Français au quotidien (1850-1950), Paris, Aubier, 1996.

14. Philippe Artières et Dominique Kalifa, «L'historien et les archives personnelles : pas à pas », Sociétés et représentations, $\mathrm{n}^{\circ} 13$, avril 2002, p. 7-15.

15. Le fonds de l'APA est situé à La Grenette, 10, rue Amédée Bonnet, 01500 Ambérieu-en-Bugey (grenette@wanadoo.fr). J'ai analysé 247 textes inédits de l'APA (191 récits autobiographiques écrits, 15 récits oraux transcrits, 32 journaux intimes, 9 correspondances). Ces textes sont l'œuvre de 216 auteurs (112 femmes et 104 hommes), nés pour près de la moitié entre 1919 et 1939 , et issus de tous les milieux sociaux.

16. Philippe Lejeune, Le Pacte autobiographique [1975], Paris, Seuil, 1996, p. 44 ; L'autobiographie en France, Paris, Armand Colin, 1998 ; «Pour l'autobiographie », Magazine littéraire, $\mathrm{n}^{\circ} 409$, mai 2002, p. 22 ; Signes de vie. Le pacte autobiographique 2, Paris, Seuil, 2005.

17. Françoise Simonet-Tenant, Le Journal intime, Paris, Nathan, 2001, p. 12 ; Philippe Lejeune et Catherine Bogaert, Un journal à soi. Histoire d'une pratique, Paris, Textuel, 2003, p. 9.

18. Roger Kempf, L'Indiscrétion des frères Goncourt, Paris, Grasset, 2004.

19. J'ai étudié une partie de ces correspondances. Le fonds Menie Grégoire a également été exploité par Anne-Marie Sohn, Âge tendre et tête de bois. Histoire des jeunes des années I960, Paris, Hachette, 2001 et «Les individus-femmes entre négation du moi et narcissisme. Les auditrices de Menie Grégoire (1967-1968) », dans Geneviève Dreyfus-Armand, Robert Frank, Marie-Françoise Lévy et Michelle Zancarini-Fournel (dir.), Les Années 68. Le temps de la contestation, Paris, Complexe, 2000, p.179-197; Marie-Véronique Gauthier, Le Cœur et le Corps. Du masculin dans les années soixante. Des hommes écrivent à Menie Grégoire, Paris, Éditions Imago, 1999.

20. Cécile Dauphin, «Les correspondances comme objet historique : un travail sur les limites ", Sociétés et représentations, $\mathrm{n}^{\circ} 13$, avril 2002, p. 47-48. Selon l'historienne, les lettres renseignent avant tout sur un imaginaire amoureux, à travers des codes sociaux ou rhétoriques.

21. Philippe Lejeune, "Je ne suis pas une source ", Sociétés et représentations, $\mathrm{n}^{\circ} 13$, avril 2002 , p. 90.

22. Philippe Artières et Dominique Kalifa, «L'historien et les archives personnelles : pas à pas », op. cit., p. 11.

23. Voir Martine Sevegrand, L'Amour en toutes lettres. Questions à l'abbé Viollet sur la sexualité, 1924-1943, Paris, Albin Michel, 1996.

24. Expression de Françoise Thébaud, «La peur au ventre », dans Georges Duby et Philippe Ariès (dir), Amour et sexualité en Occident, Paris, Seuil, coll. « Points Histoire », 1991, p. 285-297.

25. Du moins dans celles de l'APA. Il existe cependant de nombreux textes de célébrités ayant des pratiques homosexuelles dans l'entre-deux-guerres. Cf. Florence Tamagne, Histoire de l'homosexualité en Europe : Berlin, Londres, Paris, 1919-1939, Paris, Seuil, coll. «L'univers historique », 2000.

26. Idée proposée par Robert A. Nye, "Sex and Sexuality in France since 1800 ", dans Franz X. Eder, Lesley A. Hall et Gert Hekma (eds.), Sexual Culture in Europe, t. I, Manchester, Manchester University Press, 1999, p. 93.

27. Cyril Olivier, Le Vice ou la Vertu. Vichy et les politiques de la sexualité, Toulouse, Presses universitaires du Mirail, 2005.

28. Lire à ce sujet Anne-Claire Rebreyend, "Perturbation dans le genre ? Une histoire d'amour sous Vichy", dans Régis Revenin (dir.), Hommes et masculinités de 1789 à nos jours. Contributions à l'histoire du genre et de la sexualité en France, Paris, Autrement, 2007, p. 148-162.

29. Pour un aperçu de témoignages féminins, consulter Jacqueline Deroy et Françoise-Renée Pineau, Celles qui attendaient témoignent aujourd'hui, Melun, ANRPAPG, 1985.

30. D’après Kristin Ross, Aller plus vite, laver plus blanc. La culture française au tournant des années soixante [1995], Paris, Abbeville, 1997, p. 17. 
31. Voir à ce sujet, Anne-Claire Rebreyend, «Les filles "amoureuses": une nouvelle catégorie dans la France des années 1950 ", dans Véronique Blanchard, Régis Revenin et Jean-Jacques Yvorel (dir.), Jeunes, jeunesses et sexualités, Autrement, à paraître en 2009.

32. Voir Geneviève Dreyfus-Armand, Robert Frank, Marie-Françoise Lévy, et Michelle ZancariniFournel (dir.), op.cit. et Philippe Artières et Michelle Zancarini-Fournel (dir.), 68. Une histoire collective (1962-1981), Paris, La Découverte, 2008.

33. Michel Bozon, Sociologie de la sexualité, Paris, Nathan, 2002; «Révolution sexuelle ou individualisation de la sexualité ?», Mouvements, $\mathrm{n}^{\circ} 20$, mars-avril 2002, p. 15-22 ; «Les cadres sociaux de la sexualité », Sociétés contemporaines, $n^{\circ} 41-42,2001$, p. 5-9.

34. Georges Vigarello, Histoire de la beauté. Le corps et l'art d'embellir de la Renaissance à nos jours, Paris, Seuil, coll. « L'univers historique » 2004, p. 252.

35. David Le Breton, Anthropologie du corps et modernité, Paris, PUF, 1990 et La Sociologie du corps, Paris, PUF, 1992.

36. Pour un développement de cette idée, Anne-Claire Rebreyend, " "Suis-je normal(e) ?" L'obsession de la normalité sexuelle (France 1965-1975)», dans Régine Beauthier, Valérie Piette et Barbara Truffin (dir.), Révolutions sexuelles, Éditions de l'Université de Bruxelles, à paraître en 2009.

37. D'après l'enquête du docteur Pierre Simon datant de 1972.

38. Alain Giami, «Santé sexuelle : la médicalisation de la sexualité et du bien-être », dans Ruwen Ogier et Jean-Cassien Billier (dir.), Comprendre la sexualité, Paris, PUF, 2005, p. 104.

39. Jean-François Sirinelli, «La France des sixties revisitée », Vingtième siècle. Revue d'histoire, $\mathrm{n}^{\circ} 69$, janvier-mars 2001, p. 121.

40. Notons que l'anthropologie est pionnière dans ce domaine (Malinowski et Mead dès l'entredeux-guerres, puis Lévi-Strauss en 1949). La sexualité devient plus tardivement un sujet d'étude chez les sociologues (Dominique Wolton en 1974, André Béjin et Michael Pollak en 1977) et les historiens (Jean-Louis Flandrin en 1975 et 1976, Michel Foucault en 1976, Alain Corbin, Jean-Paul Aron et Roger Kempf en 1978).

\section{RÉSUMÉS}

Cet article aborde la question de la façon dont on peut écrire une histoire de l'intimité, en se concentrant sur le $\mathrm{xx}^{\mathrm{e}}$ siècle et en se basant sur de nouvelles sources: les archives autobiographiques (les journaux intimes, les histoires autobiographiques et la correspondance). Comment les individus ont-ils imaginé leurs pratiques amoureuses et quel sens avaient-elles ? Le domaine de l'intimité reste en retrait pendant la période de l'entre-deux-guerres, il est remis en question entre 1939 et 1965 et se retrouve finalement exposé au grand jour pendant la période de mai 1968 (de 1965 à 1975).

This article addresses the question as how to write a history of intimacy, focusing on the twentieth century and based on new sources: autobiographical archives (personal diaries, autobiographical stories and correspondence). How did individuals imagine their amorous practices and what meaning did they hold? The realm of intimacy remains subdued during the interwar period, challenged during 1939-1965 and finally brought out into the open during the May' 68 period (1965-1975). 
INDEX

Mots-clés : histoire de l'intimité, amour, sexualité, archives autobiographiques, vingtième siècle

Keywords : history of intimacy, love, sexuality, autobiographical archives, twentieth century

\section{AUTEUR}

ANNE-CLAIRE REBREYEND

Collège français de Reus (Espagne) 